\title{
Electronic states and magnetotransport in quantum waveguides with nonuniform magnetic fields
}

\author{
Ben-Yuan Gu \\ CCAST (World Laboratory), P.O. Box 8730, Beijing 100080, China \\ and Institute of Physics, Academia Sinica, P.O. Box 603, Beijing 100080, China* \\ Wei-Dong Sheng \\ Institute of Semiconductors, Academia Sinica, P.O. Box 912, Beijing 100083, China \\ Xue-Hua Wang \\ CCAST (World Laboratory), P.O. Box 8730, Beijing 100080, China \\ and Institute of Physics, Academia Sinica, P.O. Box 603, Beijing 100080, China \\ Jian Wang \\ Department of Physics, The University of Hong Kong, Pokfulam Road, Hong Kong, China
}

(Received 4 June 1997; revised manuscript received 26 August 1997)

\begin{abstract}
The electronic states and magnetotransport properties of quantum waveguides (QW's) in the presence of nonuniform magnetic fields perpendicular to the QW plane are investigated theoretically. It is found that the magnetoconductance of those structures as a function of Fermi energy exhibits stepwise variation or squarewave-like oscillations, depending on the specific distributions (both in magnitude and direction) of nonuniform magnetic fields in QW's. We have investigated the dual magnetic strip structures and three magnetic strip structures. The character of the magnetotransport is closely related to the effective magnetic potential and the energy-dispersion spectrum of electron in the structures. It is found that dispersion relations seem to be combined by different sets of dispersion curves that belong to different individual magnetic subwaveguides. The magnetic effective potential leads to the coupling of states and the substantial distortion of the original dispersion curves at the interfaces in which the abrupt change of magnetic fields appears. Magnetic scattering states are created. Only in some three magnetic strip structures, these scattering states produce the dispersion relations with oscillation structures superimposed on the bulk Landau levels. It is the oscillatory behavior in dispersions that leads to the occurrence of square-wave-like modulations in conductance. [S0163-1829(97)08844-9]
\end{abstract}

\section{INTRODUCTION}

The character of electron transport in microstructures created by high-mobility $\mathrm{GaAs}-\mathrm{Al}_{x} \mathrm{Ga}_{1-x} \mathrm{As}$ heterostructures in a perpendicular magnetic field has become an extremely extensive research subject of great theoretical and experimental interest. ${ }^{1}$ The prospect of building devices based on the electron coherent transport feature is an exciting area of semiconductor and device research. One important lowdimensional device is the electron waveguide, which can be achieved via field effects on a two-dimensional electron gas (2DEG) system. ${ }^{2,3}$ Another important quantum interference device is dual-coupled quantum waveguides, which can behave as an electron waveguide coupler. ${ }^{4-11}$ Electrontransport properties in nonuniform magnetic fields have also attracted much attention and are now under intensive investigations in recent years. ${ }^{12-20}$

Recently, Müller studied the single-particle electronic structure of a $2 \mathrm{DEG}$ in the wide quantum waveguide under the application of a nonuniform magnetic field $B .{ }^{12} \mathrm{He}$ found that in the presence of laterally linearly varying magnetic field in the interior of the sample the Landau states are no longer stationary but propagate perpendicularly to the field gradient and exhibit a remarkable time-reversal asymmetry. In one of the allowed directions the propagation has free- electron character, and it is confined to a narrow onedimensional region localized near the line where the magnetic field is minimum. Transport properties of electrons become one dimensional. In the the opposite direction the Landau states propagate throughout the rest of the sample with a velocity that depends on the field gradient, i.e., electrons are drifting in the interior of the sample.

More recently, Chklovskii studied the structure of the 2DEG edge in the quantum Hall regime using the compositefermion approach. ${ }^{13}$ When assuming that the electron density roughly follows the positive background and hence the composite fermions experience a roughly linearly varying effective magnetic field, there are three types of classical orbits for composite fermions in this field: $:^{13,12,21}$ drifting orbits that move along the edge in the direction of electron drift, snake orbits that move in the opposite direction, and closed orbits that do not drift along the edge.

In the previous work, ${ }^{20}$ we have theoretically investigated the magnetotransport properties of dual-coupled quantum wires in uniform magnetic fields, in which two quasi-onedimensional quantum wires are coupled through a thin isolating potential barrier. The dispersion curves exhibit an oscillation structure superimposed on the bulk Landau levels, owing to the energy splits at the cross points of two dispersion curves that belong to different quantum wires. That 
leads to the appearance of square-wave modulation in conductance.

Motivated by these works, in this paper we investigate the characters of electronic states and the magnetotransport in the quantum waveguides in nonuniform magnetic fields that take a form of laterally segmented uniformity, i.e., abruptly changing the magnitude or polarity of magnetic fields between the adjacent magnetic channels. It is believed that the interfaces, at which the abrupt change of magnetic fields takes place, play a similar role of the isolating barrier inside the waveguides. The lateral stepwise varying magnetic fields, such as dual magnetic strip and three magnetic strip structures, partition the waveguide into several parallel channels. Due to the coupling effect of electronic states between adjacent channels the magnetoconductance as a function of Fermi energy demonstrates a quantization change in a simple staircase fashion or square-wave-like oscillations, depending sensitively on the type of modulations, the magnitude, and the relative polarity of the magnetic fields. To understand this feature, we have calculated the effective potential induced by the magnetic field and the energy-dispersion relations of the propagating modes. It is found that dispersion relations seem to be combined by different sets of dispersion curves that originally belong to individual magnetic channels. However, owing to the drastic change of magnetic fields at interfaces the strong magnetic scattering takes place and leads to significant distortion of the dispersions at cross points where two sets of original dispersion curves intersect. The appearance of magnetic scattering states remarkably modifies the behavior of the dispersions of electrons in the structures. Our calculations show that in some threemagnetic-strip structures the coupling effect gives rise to the dispersions with oscillation structures superimposed on the bulk Landau levels. It is this oscillatory behavior in the dispersions that causes the square-wave-like modulations in the magnetoconductance spectrum.

This paper is organized as follows: Section II is a brief description of the model device structure and the necessary formulas used in calculations. The calculated results are presented in Sec. III with analyses. Finally, a brief summary is reserved for Sec. IV.

\section{MODEL AND FORMULAS}

The system considered is a long narrow quantum waveguide (QW) of a width $W$ subjected to a nonuniform magnetic field perpendicular to the QW lying in the $X-Y$ plane. The nonuniform magnetic-field distribution takes a simple stepwise variation in magnitude or polarity of magnetic fields, such as dual-magnetic-strip-like or three-magneticstrip-like structures, and partitions the QW into several parallel channels, in each of them the magnetic field is constant. An experimental realization of our model devices may be achieved by depositing several strips of magnetic or superconducting material on top of a 2DEG, say, on the surface of $\mathrm{GaAs} / \mathrm{Al}_{x} \mathrm{Ga}_{1-x} \mathrm{As}$ heterostructures. The adjacent channels are coupled to each other through the interface showing the abrupt change of magnetic fields. We choose, for simplicity of calculation, the hard-wall confinement potential for the boundaries. The transverse potential inside the $\mathrm{QW}$ is set to zero, i.e.,

$$
V_{c}(y)= \begin{cases}0, & 0 \leqslant y \leqslant W \\ \infty & \text { otherwise. }\end{cases}
$$

A magnetic field with magnitude $B(y)$ is applied in the $\hat{\mathbf{z}}$ direction. In the effective electron mass approximation and choosing the Landau gauge for the vector potential of $\mathbf{A}=\left[-A_{0}(y), 0,0\right]$ and $B_{z}(y)=d A_{0}(y) / d y$, the wave function of the Schrödinger equation of a single electron can be expressed as

$$
\Psi(x, y)=e^{+i k_{x} x} \psi(y),
$$

where $\psi(y)$ satisfies the one-dimensional Schrödinger equation

$$
\begin{aligned}
& \left(-\frac{\hbar^{2}}{2 m^{*}} \frac{d^{2}}{d y^{2}}+\frac{\hbar^{2}}{2 m^{*}}\left[k_{x}-e A_{0}(y) / \hbar c\right]^{2}+V_{c}(y)\right) \psi(y) \\
& =E \psi(y) .
\end{aligned}
$$

Here, $m^{*}=0.067 m_{0}$ is the isotropic effective mass for GaAs. The magnetic field enters in the Scrödinger equation as an additional momentum proportional to the vector potential. For the convenience of the following discussions, we introduce the effective potential incorporating the effect of the magnetic field as $V_{\text {eff }}(y)$ and Eq. (2a) becomes

$$
\left(-\frac{\hbar^{2}}{2 m^{*}} \frac{d^{2}}{d y^{2}}+V_{\mathrm{eff}}(y)+V_{c}(y)\right) \psi(y)=E \psi(y),
$$

where $V_{\text {eff }}(y)$ is defined by

$$
V_{\mathrm{eff}}(y)=\frac{\hbar^{2}}{2 m^{*}}\left[k_{x}-e A_{0}(y) / \hbar c\right]^{2} .
$$

The total potential is $V(y)=V_{\mathrm{eff}}(y)+V_{c}(y)$. To solve this equation, we expand $\psi(y)$ in terms of a set of complete bases, corresponding to the transverse eigenfunctions of the QW at the zero field,

$$
\psi(y)=\sum_{j=1}^{N_{s}} f_{j}(y) c_{j}
$$

where

$$
f_{j}(y)=\sqrt{\frac{2}{W}} \sin \left(\frac{\pi j}{W} y\right),
$$

and $c_{j}$ is determined by

$$
\begin{aligned}
& \sum_{j=1}^{N_{s}}\left\{\left[\left(\frac{k_{F} W}{\pi}\right)^{2}-j^{2}\right] \delta_{n j}\right. \\
& \left.\quad-\left\langle n\left|\left[k_{x} /\left(\frac{\pi}{W}\right)-2 A_{0}(y) W / \phi_{0}\right]^{2}\right| j\right\rangle\right\} c_{j}=0,
\end{aligned}
$$

where $k_{F}^{2}=2 m^{*} E_{F} / \hbar^{2}, \phi_{0}=h c / e$ is the quantum flux, and

$$
\langle n|G(y)| j\rangle \equiv \int_{0}^{W} f_{n}(y) G(y) f_{j}(y) d y .
$$


(a)
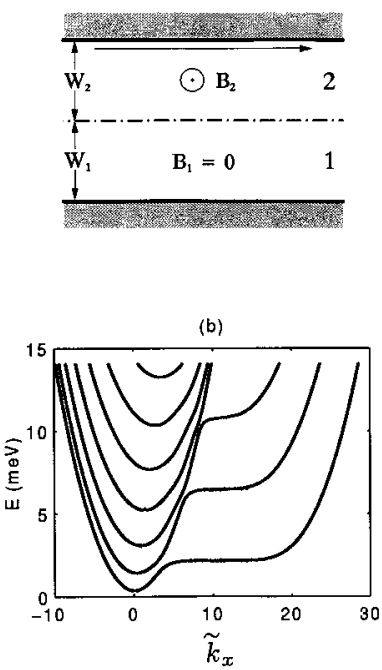

(c)

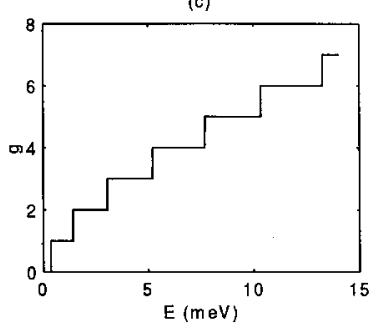

(d)

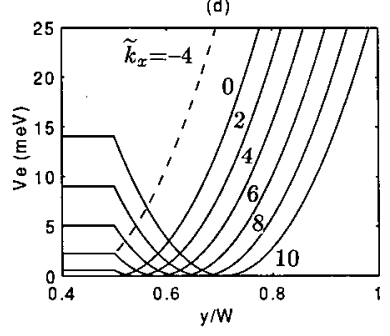

(a)

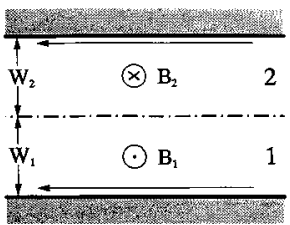

(b)

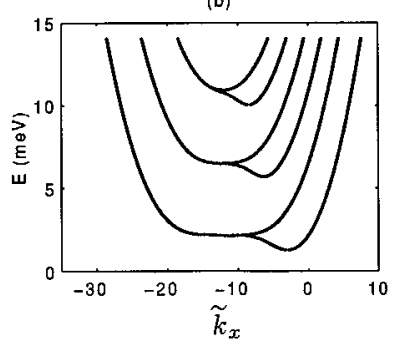

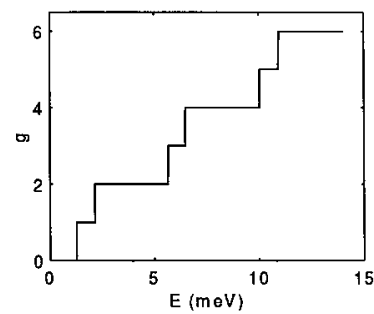

(d)

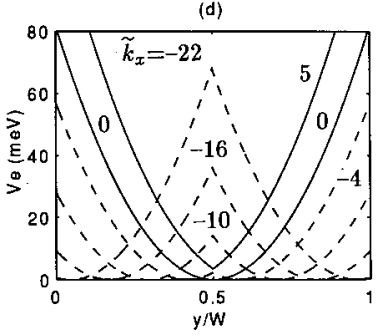

FIG. 1. Results for the QW with dual-magnetic-strip modulation structure. (a) Schematic view of a model device. The dual magnetic strips partition the QW into two parallel SQW's; in each of them the perpendicular magnetic field (in the $\hat{z}$ or $-\hat{z}$ direction) is constant. The widths of the SQW's are $W_{1}$ and $W_{2}$, respectively. Total width of the QW is $W=W_{1}+W_{2}$. The magnetic fields are $B_{1}$ and $B_{2}$ for SQW1 and SQW2, respectively. The currents carried by edge states are schematically shown by the arrow line. Parameters are as follows: $W=2000 \AA, W_{1}=W_{2}=1000 \AA, B_{1}=0$, and $B_{2}$ $=2.5 \mathrm{~T}$. (b) Energy-dispersion relation of electron [the reduced wave number $\left.\widetilde{k_{x}}=k_{x} /(\pi / W)\right]$. (c) Calculated conductance (in units of $2 e^{2} / h$ ) as a function of Fermi energy. (d) Magnetic effective potential.

Hereafter we always employ dimensionless quantities. The energy is measured in units of $E_{1}=\left(\hbar^{2} / 2 m^{*}\right)(\pi / W)^{2}$; the length is measured by $W$. In the dimensionless representation the effective potential of Eq. (3a) can be expressed by

$$
V_{\text {eff }}(\widetilde{y})=E_{1}\left[\widetilde{k_{x}}-2 A_{0}(\widetilde{y}) W^{2} / \phi_{0}\right]^{2},
$$

where $\widetilde{k_{x}}=k_{x} /(\pi / W)$ and $\tilde{y}=y / W$. It is worth pointing out that this effective potential possesses the following scaling invariance: When $W \rightarrow \alpha W$ and at the same time $B \rightarrow B / \alpha^{2}$ the normalized effective potential remains unchanged. Equation (5) can be solved in an expanded basis. ${ }^{22,23}$ For a given energy $E_{F}$, we obtain a set of eigen-wave-numbers $\left\{ \pm k_{x, n}\right\}$ and eigen-wave-functions $\left\{\psi_{n}^{ \pm}(y)\right\}$.

Assuming that two reservoirs connecting to the QW fill all the states below the Fermi energy completely and the QW is long enough so that there is no backscattering process related to the end of $\mathrm{QW}$, then, the magnetoconductance of the structures is given by ${ }^{24}$

$$
G\left(E_{F}\right)=\left(2 e^{2} / h\right) N_{p}\left(E_{F}\right),
$$

where $N_{p}\left(E_{F}\right)$ stands for the number of propagating modes with positive group velocity at the Fermi energy $E_{F}$. The group velocity of an electron in the propagating mode $\psi_{\alpha}$ is evaluated with

FIG. 2. Results for the QW with dual-magnetic-strip modulation structure. (a) Schematic view of the model device. The geometrical structure and relevant parameters are the same as Fig. 1(a) except for $B_{1}=2.5 \mathrm{~T}$ and $B_{2}=-2.5 \mathrm{~T}$. (b) Energy-dispersion curves. (c) Calculated conductance curves. (d) Magnetic effective potential.

$$
v_{\alpha}=\frac{\hbar}{m^{*}} \int_{0}^{W} \psi_{\alpha}^{*}\left(k_{\alpha}-\frac{2 \pi A_{0}(y)}{\phi_{0}}\right) \psi_{\alpha}(y) d y .
$$

\section{RESULTS AND ANALYSES}

We now calculate the energy spectrum and the magnetoconductance of the system where we have fixed the width of the QW to be $W=2000 \AA$. First, we consider the dualmagnetic-strip structures. The relevant vector potential takes the form

$$
A_{0}(y)=\left\{\begin{array}{cc}
B_{2}\left(y-W_{1}\right)+B_{1}\left(W_{1}-W / 2\right), & W_{1} \leqslant y \leqslant W \\
B_{1}(y-W / 2), & 0 \leqslant y<W_{1} .
\end{array}\right.
$$

The schematic view of the typical model devices are shown in Figs. 1(a) and 2(a). The dual magnetic strips partition the QW into two parallel sub-wave-guides (SQW's), in each of them the perpendicular magnetic field is constant. The widths of the SQW's are $W_{1}$ and $W_{2}$, respectively, and $W_{1}$ $=W_{2}=W / 2=1000 \AA$. The magnetic fields are denoted as $B_{1}$ and $B_{2}$ for SQW1 and SQW2, respectively. The currents carried by edge states are indicated by the arrow lines. We consider two cases: one is $B_{1}=0$ and $B_{2}=2.5 \mathrm{~T}$ [see Fig. 1(a)], the other is $B_{1}=+2.5 \mathrm{~T}$ and $B_{2}=-2.5 \mathrm{~T}$ [see Fig. 2(a)]. The corresponding energy dispersion relations of electrons are displayed in Figs. 1(b) and 2(b), respectively. The corresponding conductances as a function of Fermi energy of electrons are plotted in Figs. 1(c) and 2(c). It is seen from Fig. 1(b) that the energy spectrum is constructed by the combination of two sets of dispersion curves separated by a distance in wave-number space. One set of dispersion curves is parabolic, located around the origin of $\widetilde{k_{x}}=0$, belonging to the SQW1 with $B_{1}=0$. Only bulk propagating modes exist. 
The other set of curves belongs to the SQW2 with $B_{2}$ $=2.5 \mathrm{~T}$, in which there exists both bulk Landau levels and edge states located at the upper boundary (monotonically increasing behavior in dispersion). Two sets of dispersion curves intersect each other to create cross points. Since the abrupt change of the magnetic fields at the interface of two SQW's, the coupling effect between states takes place. It leads to the creation of the magnetic scattering states ${ }^{12,13}$ and substantially distorts the behavior of dispersions around the cross points, as seen in Fig. 1(b). Figure 1(c) shows the calculated conductance of this QW as a function of the Fermi energy. The conductance steps up normally in the wellknown staircase fashion with integer conductance plateaus in units of $2 e^{2} / h$. This character can be well understood from the energy dispersion of the QW. As the Fermi energy of electron increases, additional propagating channels are opened. Thus, the conductance is increased stepwise.

To give a better understanding on the character of dispersion curves, we display the profile of effective potential $V_{\text {eff }}(y)$ for several values of $\widetilde{k_{x}}$ in Fig. 1(d). For above chosen parameters of the model device, the corresponding effective potential, Eq. (3b), reads

$$
V_{\text {eff }}(y)=\left\{\begin{array}{cc}
E_{1}\left(\widetilde{k_{x}}\right)^{2}, & 0 \leqslant y / W<0.5 \\
E_{1}\left[\widetilde{k_{x}}-\beta(y / W-0.5)\right]^{2}, & 0.5 \leqslant y / W \leqslant 1,
\end{array}\right.
$$

where $\beta=2 B_{2} W^{2} / \phi_{0}$. Thus, in the SQW1, $V_{\text {eff }}$ is constant and its value increases quadratically with $\widetilde{k}_{x}$. In the SQW2 region, when $\widetilde{k_{x}} \leqslant 0, V_{\text {eff }}$ takes the form of $E_{1} \beta^{2}(y / W-0.5$ $\left.+\left|\widetilde{k}_{x}\right| / \beta\right)^{2}$ and it is a monotonically increasing function. Thus, electron propagation in the SQW1 has a free-electron feature, and, therefore, the corresponding energy dispersion should be parabolic in shape when $\widetilde{k_{x}} \leqslant 0$. However, for $\widetilde{k_{x}}$ $>0, V_{\text {eff }}$ exhibits a modified parabolic well potential with a finite height at the left edge of the well owing to zero field in the SQW1. The depth of the well is increased quadratically with $\widetilde{k}_{x}$ and the position $y_{0} / W$ of the well bottom shifts toward the upper boundary of the SQW2 as increasing $\widetilde{k_{x}}$, i.e., $y_{0} / W=0.5+\widetilde{k_{x}} / \beta$. The oscillating frequency of the harmonic oscillator is constant proportional to $B_{2} W^{2}$, independent of $\widetilde{k}_{x}$. It is the existence of the modified parabolic well that leads to the emergence of the Landau levels in the region of $\widetilde{k_{x}}>0$. For small $\widetilde{k_{x}}$, the well is quite shallow, consequently, there is no Landau level and electrons are propagating almost freely. The corresponding dispersion curve is parabolic. When increasing $\widetilde{k_{x}}$ to a certain value, the modified parabolic well becomes deep enough to lead to the occurrence of the first Landau level. Thus, the first Landau plateau emerges. As continuously increasing $\widetilde{k_{x}}$, the well is deepened, as a result, more Landau levels are formed in the well. This is the reason why the position of kink points from the parabolic dispersion to the Landau plateau in dispersion curves shifts toward the larger wave-number region for larger Landau index, as shown in Fig. 1(b). Of course, the rapidly monotonically increasing behavior in dispersions for much larger $\widetilde{k_{x}}$ is originated from the contribution of the edge states of the upper boundary of SQW2. Owing to the parabolic well bottom gradually shifting toward the bound- ary of SQW2 with the increase of $\widetilde{k_{x}}$, the width of the paraboliclike well becomes narrower and total potential well, incorporating the confinement potential, develops a deep triangular well. Therefore, the Landau levels with large indices are completely squeezed out from the well; thus, the width of the Landau plateaus is shortened with the increase of the Landau-level index. We now can perfectly understand all the behaviors of dispersion curves in Fig. 1(b).

We now discuss the second model device, as shown in Fig. 2(a). Two SQW's are now experienced to the magnetic fields with the same magnitude in absolute value and opposite polarity: $B_{1}=+2.5 \mathrm{~T}$ and $B_{2}=-2.5 \mathrm{~T}$. The corresponding energy dispersions are depicted in Fig. 2(b). The lines in the left branch of each subband are contributed from the left-moving edge states located, respectively, at the uppermost and lowermost boundaries, as shown in Fig. 2(a), with twofold degeneracy. The coupling between them is absent due to their large separation in space. However, two lines of each subband in the right branch of dispersions are ascribed to the contribution of magnetic scattering states owing to the abrupt reversal of polarity of the magnetic fields at the interface. That is the reason why the dispersion behavior exhibits high asymmetry in wave-number space owing to different origins. For different $B_{1}$ and $B_{2}$, for instance, $B_{1}=2.75 \mathrm{~T}$ and $B_{2}=-2.5 \mathrm{~T}$, we found that all the left branches of the dispersion curves split into two lines grouped in pairs. By simply enumerating the number of propagating modes at the Fermi level the conductance can be evaluated as a function of Fermi energy, as shown in Fig. 2(c). The stepwise variation still persists but the width of plateaus changes alternatively between narrow and broad steps.

To get a better understanding of the dispersion behavior in Fig. 2(b), we now study the profile of the effective potential. The results are shown in Fig. 2(d) for several values of $\widetilde{k_{x}}$. It is clearly seen that the trianglelike potential barrier is produced around the interfacial region between two SQW's when $\widetilde{k_{x}}<0$. When $\widetilde{k_{x}} \geqslant 0$, however, this barrier completely disappears and is replaced by a potential well. The height of the triangular barrier rapidly decreases with the decrease of $\left|\widetilde{k_{x}}\right|$. The total potential exhibits a coupled double-quantumwell structure with a triangular barrier of finite height. The energy levels in the trianglelike quantum wells contribute to the left branch of the dispersions. When $\widetilde{k}_{x} \leqslant-10$, the height of the triangle barrier is so low that the coupling between two quantum wells becomes strong, thus, the split of two equal energy levels in the wells appears, leading to the "bifurcation" of subbands in energy dispersion spectrum. When $\widetilde{k}_{x}$ is zero, the triangular barrier reduces to zero, and the double wells becomes a single well, as shown in Fig. 2(d). For $\widetilde{k}_{x}>0$, the effective potential remains the singlequantum-well structure where minimum increases with $\widetilde{k}_{x}$. It is those electron states in the single wells that contribute to the right branch of dispersions.

We now turn to envisage the character of the QW's with three magnetic strip structures. Schematic view of this kind of model devices is shown in Fig. 3(a). Three magnetic strips produce stepwise varying magnetic field in the QW with width of $W$ and partition the QW into three parallel SQW's. The widths of the SQW's are denoted as $W_{1}, W_{2}$, and $W_{3}$, respectively. The relevant vector potential takes the form 


$$
A_{0}(y)=\left\{\begin{array}{cc}
B_{3}\left(y-W_{1}-W_{2}\right)+B_{2}\left(W_{1}+W_{2}-W / 2\right), & \left(W_{1}+W_{2}\right) \leqslant y \leqslant W \\
B_{2}(y-W / 2), & W_{1} \leqslant y<\left(W_{1}+W_{2}\right) \\
B_{1}\left(y-W_{1}\right)+B_{2}\left(W_{1}-W / 2\right), & 0 \leqslant y<W_{1} .
\end{array}\right.
$$

Besides the edge states, extra magnetic scattering states are created owing to the drastic change of magnetic fields at the interfaces between two adjacent SQW's. The currents carried by the edge states are shown by the arrow lines. Parameters for the first magnetic sandwiching device are as follows: $W$ $=2000 \AA, \quad W_{1}=W_{2}=W_{3}=W / 3, B_{1}=B_{3}=2.5 \mathrm{~T}$, and $B_{2}$ $=0$. The relevant energy spectrum is displayed in Fig. 3(b). The corresponding conductance spectrum is plotted in Fig. 3(c). This conductance spectrum exhibits a trivial stepwise dependence. The energy spectrum seems to be combined by three sets of dispersion curves separated by some distances in wave-number space: one set of dispersion curves has parabolic shape, located in $\widetilde{k_{x}}=0$ region, belonging to the SQW2 with zero field, in which electron propagation has freeelectron character. The other two sets of dispersion curves are ascribed to the SQW1 and SQW3, experienced to a finite magnetic field, in which there exist both magnetic scattering states and bulk Landau levels. These three sets of dispersion curves intersect each other, producing many cross points. Owing to the overlap of wave functions and the drastic change of magnetic fields at each interface the strongcoupling effect takes place, leading to the creation of magnetic scattering states. It is the emergence of the magnetic (a)

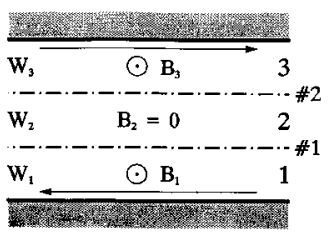

(b)

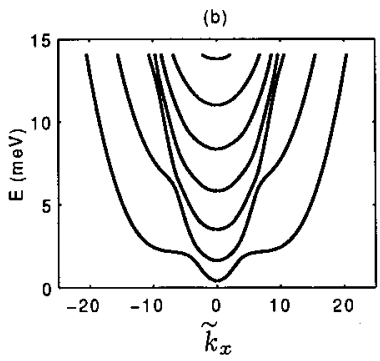

(c)

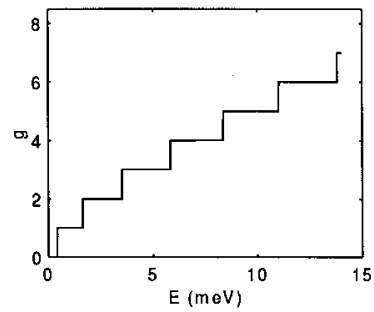

(d)

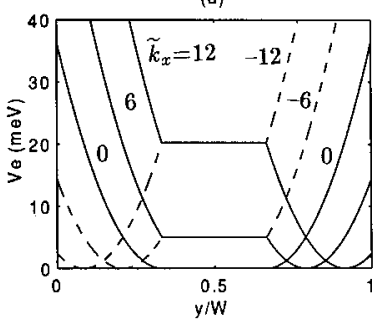

FIG. 3. Results for the QW with three-magnetic-strip modulation structure. (a) Schematic view of a model device. The threemagnetic-strips partition the QW into three parallel SQW's. The magnetic fields are $B_{1}, B_{2}$, and $B_{3}$ for three SQW's, respectively. The widths of the SQW's are $W_{1}, W_{2}$, and $W_{3}$, respectively. Total width of the QW is $W=W_{1}+W_{2}+W_{3}$. The currents carried by edge states in the SQW are schematically shown by the arrow lines. Parameters are as follows: $W=2000 \AA, W_{1}=W_{2}=W_{3}=W / 3, B_{1}$ $=B_{3}=+2.5 \mathrm{~T}$, and $B_{2}=0$. (b) Energy-dispersion relation of electron. (c) Calculated conductance as a function of Fermi energy. (d) Magnetic effective potential. scattering states that causes the remarkable distortion of the dispersion relation at cross points and the appearance of kinks. Owing to the presence of magnetic fields in SQW1 and SQW3, electron energies are increased compared with that in the SQW2 with zero field, consequently, the dispersion curves exhibit valley structures in the center region of $\widetilde{k}_{x}$. The spectral pattern is symmetric due to the complete symmetry of the structure for this device. It is clearly seen from Fig. 3(a) that the current directions for uppermost and lowermost boundary states are reversed.

To understand the dispersion relation of Fig. 3(b), we calculate the magnetic effective potential in this QW for several $\widetilde{k_{x}}$, as shown in Fig. 3(d). It is clearly seen that the profile of the potential achieves space-reversal symmetry with respect to the central line of the QW when changing the sign of $\widetilde{k_{x}}$. For the finite values of $\widetilde{k}_{x}$, all the potentials exhibit stepped quantum-well structures. The potential plateaus are located at the center of the device and their heights are increased with $\left|\widetilde{k}_{x}\right|$. In the plateau region of the potential, electrons can travel freely and the corresponding dispersions are parabolic. For $\widetilde{k_{x}}>0$, the relevant quantum wells locate inside the SQW3 with finite height in the left edge of the wells. It is these modified parabolic wells that lead to the formation of the Landau levels in the dispersions. The larger the $\widetilde{k}_{x}$, the deeper the depth of the parabolic well, thus, more Landau levels in the well. This is the reason why the kink position of the second subband in the right branch of dispersion curves shifts to a large value of $\widetilde{k_{x}}$ compared with that of the first subband. Owing to the bottom position of the potential wells shifting toward the upper boundary of SQW3 with the increase of $\widetilde{k_{x}}$, for much larger $\widetilde{k_{x}}$, the modified parabolic well approaches the upper boundary of the SQW3, and becomes a triangular well when incorporating the hard-wall confined potential. The triangular well with narrow width squeezes the Landau levels out of it, leading to the disappearance of the Landau levels with large index. This is the reason why there is no longer kink in dispersions of third subband and above. Similarly, we can interpret the behavior of the left branch of dispersion curves.

To further reveal the character of the magnetic sandwiching structural device, we investigate other model devices in detail. We now consider a magnetic-field configuration: $B_{1}$ $=B_{3}=0$ and $B_{2}=2.5 \mathrm{~T}$, as shown in Fig. 4(a). The corresponding energy dispersions are shown in Fig. 4(b), and the resulting conductance spectrum is displayed in Fig. 4(c). The magnetic effective potential is displayed in Fig. 4(d). It is evident from Fig. 4(b) that the dispersions are combined by three sets of dispersion curves originally belonging to three individual SQW's. The left branch comes from the SQW1 with parabolic dispersion and the right branch from the SQW3 with also parabolic shape owing to the absence of magnetic fields. However, the middle of dispersion curves is 
(a)
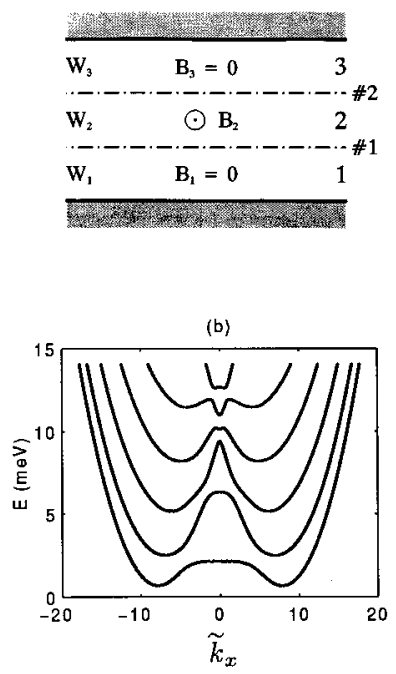

(c)

(d)

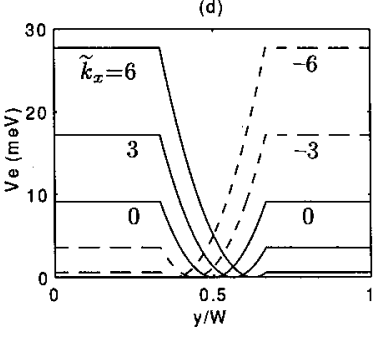

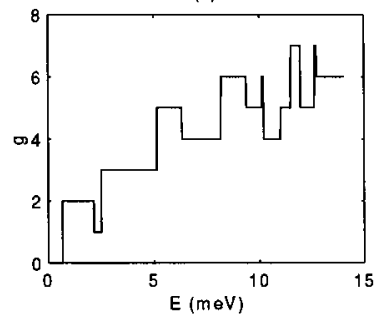

(a)
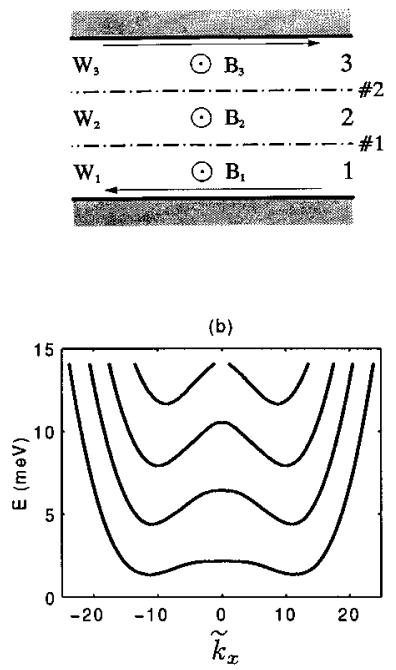

(c)

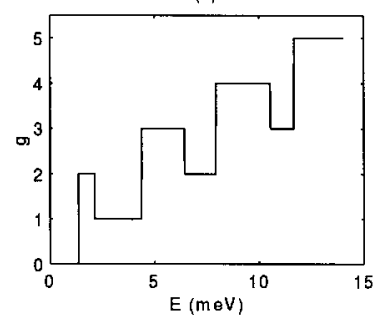

(d)

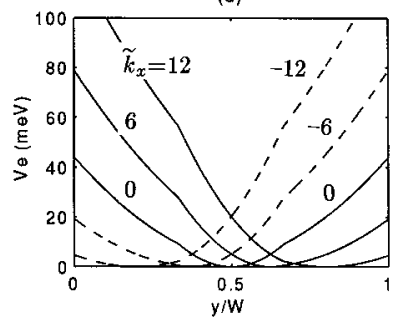

FIG. 4. Results for the QW with three-magnetic-strip modulation structure. (a) Schematic view of the model device. The geometrical structure and relevant parameters are the same as Fig. 3(a) except for $B_{1}=B_{3}=0$ and $B_{2}=+2.5 \mathrm{~T}$. (b) Energy-dispersion curves. (c) Calculated conductance curves. (d) Magnetic effective potential.

ascribed to the SQW2 with a finite field where the bulk Landau levels exist. The dispersions are symmetric due to the symmetry of the device structure. Two consecutive sets of dispersion curves are separated by a distance in wavenumber space and intersected each other. Owing to the abrupt change of magnetic fields at interface, the coupling effect takes place. It leads to the creation of the magnetic scattering states and the occurrence of the kink points in dispersions. As contrasted with the device of Fig. 3(a), the magnetic field now is applied in the middle region, i.e., in SQW2; therefore, the energy subbands in the SQW2 are lifted with respect to the zero-field regions in the SQW1 and SQW3. Finally, the bump structures in dispersion are formed in the central region of $\widetilde{k_{x}}$. At the same time, minigaps are formed. The dispersions exhibit oscillatory structures. When scanning the Fermi energy, it is these bumps and minigaps that lead to the emergence of the square-wave-shaped oscillations in conductance spectrum, as seen in Fig. 4(c). For instance, as the Fermi energy increases, additional propagating channels are opened, thus, conductance increases $2\left(2 e^{2} / h\right)$, instead of the step of $\left(2 e^{2} / h\right)$. When the Fermi energy of the electron sweeps through minigaps, conductance dips are present owing to the quenching of the propagating modes. The depth of the conductance dips depends on the number of quenched propagating modes at the given Fermi energy. As increasing the Fermi energy continuously, the new propagating channels are opened and the conductance reaches higher plateau, forming the square-wave-like conductance spectrum. The conductance spectrum consists of a series of peaks and valleys.

To provide the good explanation of the character of dispersions, we present the calculated magnetic effective potential in Fig. 4(d). It is clearly seen that the profile of the potential shows a space-reversal symmetry with respect to the central line of the model device when altering the sign of
FIG. 5. Results for the QW with three-magnetic-strip modulation structure. (a) Schematic view of the model device. Parameters are the same as Fig. 4(a) except for $B_{1}=B_{3}=1.5 \mathrm{~T}$. (b) Energydispersion relation of electron. (c) Calculated conductance as a function of Fermi energy. (d) Magnetic effective potential.

$\widetilde{k}_{x}$. The effective potential in the SQW1 (SQW3) is constant and its value increases (decreases) with $\widetilde{k_{x}}$. Electrons move freely in these regions and the corresponding dispersions are parabolic. In the SQW2 the magnetic quantum well is formed and takes a form of declined parabola with finite height in both edges. The position of the potential bottom shifts far away from the center of the QW with the increase of $\left|\widetilde{k}_{x}\right|$. The larger the $\left|\widetilde{k}_{x}\right|$, the greater the declined degree of parabolic well is. Thus, the well depth becomes much shallow, only a few of Landau levels can be found in the well. The Landau levels with large indices disappear for large $\left|\widetilde{k}_{x}\right|$. This is the reason why the width of Landau plateaus decreases as increasing the Landau subband index. The existence of Landau bumps and minigaps in dispersions guarantees the appearance of the square-wave-like modulation in conductance spectrum.

The square-wave-like modulation spectrum of conductance can be modified and improved by applying a finite field to the SQW1 and SQW3 regions. For instance, we consider the similar device as that of Fig. 4(a) except for $B_{1}=B_{3}$ $=1.5 \mathrm{~T}$. The energy dispersions, the conductance spectrum, and the magnetic effective potential are depicted in Figs. 5(b), 5(c), and 5(d), respectively. It is evident that the dispersions have similar behavior of Fig. 4(b) and the conductance exhibits much more regular square-wave-like modulation in Fig. 5(c), comparing with Fig. 4(c).

To offer more information on the appearance of squarewave-like modulation of conductance, we now study another device with sandwiching magnetic structure, as shown in Fig. 6(a). In this device, $B_{1}=B_{3}=2.5 \mathrm{~T}$ and $B_{2}=-3.0 \mathrm{~T}$. The other parameters are the same as those in Fig. 5(a). This device is composed of two blocks of dual-magnetic-strip structures of Fig. 2(a). Considering the structural symmetry on this device, it is expected that the dispersion relations should be symmetric about $\widetilde{k_{x}}=0$ point. The dispersions, the 
(a)
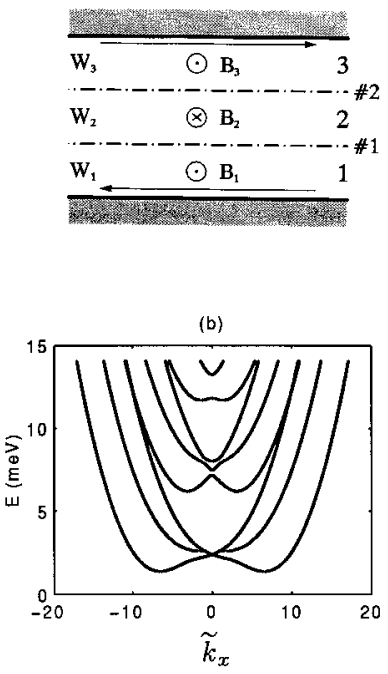

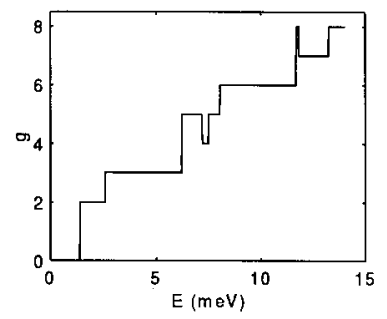

(d)

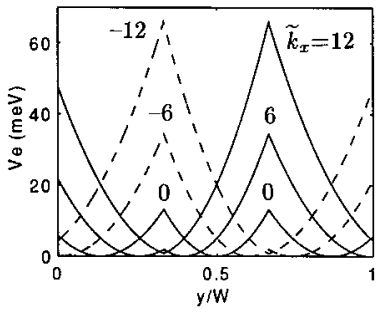

FIG. 6. Results for the QW with three-magnetic-strip modulation structure. (a) Schematic view of the model device. The geometrical structure and relevant parameters are the same as Fig. 3(a) except for $B_{2}=-3.0 \mathrm{~T}$. (e) Energy-dispersion curves. (c) Calculated conductance curves. (d) Magnetic effective potential.

conductance spectrum, and the magnetic effective potential are displayed in Figs. 6(b), 6(c), and 6(d), respectively. It is easy to specify the origin of the dispersion curves. For instance, the left branch comes from the contribution of the lowermost edge states with left-moving modes; the right branch generates from the uppermost edge states with rightmoving modes. In the central region of the dispersion curves, their behaviors are mainly determined by the property of the SQW2 and the coupling effect among the magnetic scattering states. The coupling effect leads to the substantial distortion of the dispersions. According to similar analyses as discussed above, it is expected that the conductance spectrum should consist of dips (with different depths and widths) and plateaus (with different heights and widths). The conductance spectrum exhibits the square-wave-like oscillations that we are interested in. To save space, we do not repeat similar analyses as done in the above samples. When keeping $B_{3}=2.5 \mathrm{~T}$ and $B_{2}=-3.0 \mathrm{~T}$ and setting $B_{1}=0$, similar results can be obtained but the dispersions become asymmetric in the $\widetilde{k_{x}}$ domain owing to asymmetry of the magnetic structure. So does the profile of the magnetic effective potential in the $y / W$ domain.

\section{SUMMARY}

We have studied transport properties of electrons in the QW's under the application of stepped varying magnetic fields. To reveal the nature of the magnetic scattering states owing to the abrupt change of magnetic fields at interfaces, we investigate in detail two kinds of model devices, one is dual-magnetie-strip structure, the other is three-magneticstrip structure. Magnetoconductance as a function of the Fermi energy demonstrates monotonically increasing variation in staircase or square-wave-like oscillations, consisting of plateaus and valleys. The detailed patterns of the magnetoconductance depend on the distribution of nonuniform magnetic fields. In the dual-magnetic-strip structures, the magnetoconductance spectrum always exhibits the stepwise increasing variation. To understand the origin of the conductance spectrum, we have calculated the energy-dispersion relations of the propagating states and the relevant magnetic effective potential. The stepped varying magnetic fields partition the QW into several subwaveguides; in each of them the magnetic field is constant. The whole dispersion curves can be regarded as the combination of dispersion curves of the individual SQW's. The coupling effect between SQW's leads to the creation of the magnetic scattering states, thus, the dispersions are experienced the substantial distortion at the cross points where two sets of original dispersion curves intersect each other. It causes the bend of the dispersion curves, the emergence of valleys or bumps superimposed on the flat bulk Landau levels, and the production of minigaps. Oscillatory structures in dispersions appear. It is these complex dispersions that lead to various complicated conductance patterns. The characters of the dispersions can be well interpreted by analyzing the relevant magnetic effective potential. We have presented detailed analyses for several model devices. Consequently, a new way of artificially tailoring the conductance spectrum is proposed by introducing laterally stepped varying magnetic fields perpendicular to the QW plane. It may be useful for guiding the design of new quantum devices in practice.

\section{ACKNOWLEDGMENTS}

We gratefully acknowledge the support by the National Natural Science Foundation of China and a research grant from the Croucher Foundation.
*Electronic address: guby@ aphy01.iphy.ac.cn

${ }^{1}$ C. W. J. Beenakker and H. van Houton, in Solid State Physics: Semiconductor Heterostructures and Nanostructures, edited by H. Ehrenreich and D. Turnbull (Academic, New York, 1991), Vol. 44, p. 1, and references cited therein.

${ }^{2}$ B. J. van Wees, H. van Houton, C. W. J. Beenakker, J. G. Williamson, L. P. Kouwenhoven, D. van der Marel, and C. T. Foxon, Phys. Rev. Lett. 60, 848 (1988); G. Bernstein and D. K. Ferry, J. Vac. Sci. Technol. B 5, 964 (1987).
${ }^{3}$ F. Sols, M. Macucci, U. Ravaioli, and K. Hess, J. Appl. Phys. 66, 3892 (1989).

${ }^{4}$ J. A. del Alamo and C. C. Eugster, Appl. Phys. Lett. 56, 78 (1990).

${ }^{5}$ C. C. Eugster, J. A. del Alamo, M. J. Rooks, and M. R. Melloch, Appl. Phys. Lett. 60, 642 (1992); Phys. Rev. B 46, 10146 (1992); ibid. 48, 15057 (1993); Phys. Rev. Lett. 67, 3586 (1991).

${ }^{6}$ R. Q. Yang and J. M. Xu, Phys. Rev. B 43, 1699 (1991). 
${ }^{7}$ J. Q. Wang, S. Q. Yuan, B. Y. Gu, and G. Z. Yang, Phys. Rev. B 44, 13618 (1991).

${ }^{8}$ J. Q. Wang, B. Y. Gu, and G. Z. Yang, J. Appl. Phys. 72, 2299 (1992); Phys. Rev. B 47, 13442 (1993).

${ }^{9}$ Y. Takagaki and K. Ploog, Phys. Rev. B 49, 1782 (1994).

${ }^{10}$ J. Wang, H. Guo, and R. Harris, Appl. Phys. Lett. 59, 3075 (1991).

${ }^{11}$ J. Wang, Y. J. Wang, and H. Guo, Phys. Rev. B 46, 2420 (1992); ibid. 47, 4348 (1993).

${ }^{12}$ J. E. Müller, Phys. Rev. Lett. 68, 385 (1992).

${ }^{13}$ D. B. Chklovskii, Phys. Rev. B 51, 9895 (1995).

${ }^{14}$ B. L. Altshuler and L. B. Ioffe, Phys. Rev. Lett. 69, 2979 (1992); T. Sugiyama and N. Nagoasa, ibid. 70, 1980 (1993); S.-C.
Zhang and D. P. Arovas, ibid. 72, 1886 (1994).

${ }^{15}$ Z. L. Ji and D. W. L. Sprung, Phys. Rev. B 54, 8044 (1996).

${ }^{16}$ Y. Takagaki and K. Ploog, Phys. Rev. B 51, 7017 (1995).

${ }^{17}$ Y. Takagaki and K. Ploog, Phys. Rev. B 53, 3885 (1996).

${ }^{18}$ H. Xu, Phys. Rev. B 52, 5803 (1995).

${ }^{19}$ C. S. Kim and O. Olendski, Phys. Rev. B 53, 12917 (1996).

${ }^{20}$ J. R. Shi and B. Y. Gu, Phys. Rev. B 55, 9941 (1997).

${ }^{21}$ D. K. K. Lee, J. T. Challer, and D. Y. K. Ko, Phys. Rev. B 50, 5272 (1994).

${ }^{22}$ H. Tamura and T. Ando, Phys. Rev. B 44, 1792 (1991).

${ }^{23}$ S. Chaudhuri and S. Bandyopadhyyay, J. Appl. Phys. 71, 3027 (1992).

${ }^{24}$ M. Büttiker, Phys. Rev. B 38, 9375 (1988). 